Ann. Biol. anim. Bioch. Biophys., 1977, 17 (5 A), 695-712.

\title{
La capsule testiculaire du renard roux (Vulpes vulpes $L$.) : relation avec l'activité testiculaire pendant la période prépubère et au cours du cycle saisonnier
}

par M. JOFFRE

\author{
Laboratoire de Physiologie comparée, Faculté des Sciences \\ Parc Grandmont, 37200 Tours, France \\ Centre d'Efudes Biologiques des Animaux Sauvages, \\ 79360 Beauvoir s/Niort.
}

Summary. The testicular capsule of the wild red fox (Vulpes vulpes) : relationship with testicular activity during the prepuberal period and the seasonal cycle.

Wet and dry weight total collagen content and histology of the connective structures of the testicular capsule (albuginea) and the parenchyma were studied during the prepubertal growth of the fox cub and during the adult seasonal breeding cycle.

1) Testicular capsule weight is closely related to testicular weight. It slowly increases during the impubertal period from March to September, then rapidly from October to January. A maximum value is reached at puberty in January. In the adult, this weight decreases from January to March-April ; it remains stable during the resting period, then increases again until January. All these ponderal changes are closely related to the total collagen content of the capsule. However, collagen titer, which increases during impubertal and prepubertal testis growth, does not change during the seasonal cycle of the adult.

2) Total collagen content of the parenchyma increases from March fo January in the testis of the fox cub. In the adult it decreases during the regressive period, then increases again from October to January. Collagen concentration decreases during the prepubertal period and adult re-growth period ; during the latter, the titer increases during the regressive period.

3) Connective tissue structures are mainly found in the tunica albuginea of the capsule, the septa, blood vessel and seminiferous tubule walls and the intertubular spaces in the parenchyma. In the impubertal state, tunica albuginea, septa and intertubular tissue are characterized by numerous juvenile fibroblasts and PAS-positive material (mucopolysaccharides) ; collagen fibers are not abundant. During prepubertal growth there is progressive fibrogenesis of these structures and parallel disappearance of mucopolysaccharides. At puberty, the tunica albuginea is formed by numerous coalescent fibers, some mature fibroblasts and a few smooth muscle cells. The septa contain numerous collagen fibers, and the intertubular spaces a loose connective tissue around the seminiferous tubules, the Leydig cell islands and the capillaries.

During the regressive period in the adult, the testicular capsule and septa thicken markedly, and abundant polysaccharides re-appear between the retracted collagen fibers. Simultaneously, the basal membrane of the seminiferous tubules thickens; maturation of this membrane is previously observed during the prepubertal periad of the young fox. 
These results are discussed and related to testicular activity in young and adult foxes ; the changes are related to pituitary activity. Capsular skrinkage in the adult fox during the regressive period is an active process causing spiralization of arteries and vein distortion. It appears that the testicular capsule and the septa must play a role in seasonal control of testicular hemodynamics in the adult fox.

\section{Introduction.}

L'activité génitale du renard mâle (Vulpes vulpes L.) se caractérise par un cycle saisonnier au cours duquel le testicule passe d'un état de pleine activité (période du rut) à un état, dit de « repos », et présente des variations de la spermatogenèse et de l'hormogenèse (Rowlands et Parkes, 1935 ; Creed, 1960 ; Joffre, 1976a). Parallèlement, on observe les variations du débit sanguin capillaire du testicule (Joffre et Joffre, 1973) et les modifications de la trame artérielle irriguant la glande (Joffre et Kormano, 1975).

L'existence de liens étroits entre la capsule testiculaire, plus couramment appelée albuginée, et les artères irriguant le parenchyme testiculaire permet d'envisager un rôle possible de cette enveloppe dans le contrôle de l'hémodynamique testiculaire au long du cycle génital saisonnier.

Afin de confirmer cette hypothèse, l'étude pondérale, biochimique (teneur en collagène total) et histologique de la capsule a été abordée. En outre, la période de réveil testiculaire automnal de l'adulte présentant de grandes similitudes avec la période prépubère (Joffre, 1976a), cette analyse a été poursuivie, en parallèle, chez l'animal immature et chez l'animal adulte.

\section{Matériel et méthodes.}

Animaux. - Notre étude a porté sur 166 renards mâles dont 84 immatures. Ces animaux ont été capturés de 1970 à 1974, dans les départements du Centre-Ouest et du Sud-Ouest de la France, ef mis en élevage dans les installations du Centre d'Etudes Biologiques des Animaux Sauvages. Ils ont été placés dans des cages individuelles, sous un abri leur assurant un photopériodisme naturel et une température ambiante voisine de la température extérieure. Leur nourriture était assurée par des poules mortes; de l'eau leur était donnée à volonté.

Prélèvements. - Ils ont éfé effectués sur l'animal anesthésié ou sacrifié après injection intracardiaque de nembutal. Les testicules, après avoir été pesés, ont été débarrassés de leur capsule en sectionnant les septa au ras de cette enveloppe. Chacune des composantes est pesée, puis stockée à $-25^{\circ} \mathrm{C}$ pour le dosage ultérieur du collagène total ; quelques fragments ont été fixés au Bouin-Hollande. L'état de la spermatogenèse a été contrôlé sur des coupes colorées à l'hémalunpicro-indigocarmin ou au PAS-hématoxyline et celui de la capsule, sur des coupes colorées au trichrome de Masson ou au PAS-hématoxyline. 
Testostérone plasmatique. - Les taux de testostérone plasmatiques ont été déterminés sur du sang prélevé dans la veine radiale, chez des renards impubères ou pubères anesthésiés. La testostérone a été dosée selon la technique radioimmunologique de Leymarie, Strauss et Scholler (1974), validée pour le Renard (Joffre et Joffre, 1975).

Collagène total. - Le parenchyme et la capsule testiculaire ont été traités séparément selon la technique de Neuman et Logan (1950), appliquée au testicule de Rat par Denduchis et Mancini (1967).

Les tissus, délipidés à l'acétone puis à l'éther 3 fois par jour pendant 3 jours, sont déshydratés à l'étuve, pendant $24 \mathrm{~h}$ à $108^{\circ} \mathrm{C}$. Le collagène est alors solubilisé en milieu aqueux, sous une pression de 1 atm., pendant 3 h. Après lavage à l'eau, l'opération est répétée. L'ensemble des surnageants est ensuite évaporé à sec, puis repris par $\mathrm{HCl}$ $(6 \mathrm{~N})$. L'hydrolyse du collagène est réalisée en tube bouché, à $104^{\circ} \mathrm{C}$ pendant $24 \mathrm{~h}$. Le système est enfin neutralisé à la soude.

Le dosage de l'hydroxyproline est réalisé sur les hydrolysats selon la technique photocolorimétrique de Neuman et Logan (1950), modifiée par Martin et Axelrod (1963) ef Blomfield et Farrar (1964). L'acide aminé est oxydé en tube bouché par de l'eau oxygénée à 6 p. 100 , en milieu alcalın, en présence de sulfate de cuivre $(0,05 \mathrm{M})$, pendant $10 \mathrm{mn}$ à $40^{\circ} \mathrm{C}$. Une solution de p-dimethylaminobenzaldéhyde à $3 \mathrm{p} .100$ dans de l'alcool propylique à 40 p. 100 est ensuite ajoutée. La réaction s'effectue en tube bouché à $70^{\circ} \mathrm{C}$ pendant $42 \mathrm{mn}$, temps au bout duquel on stabilise la coloration par de l'alcool propylique froid. Le produit de la réaction est mesuré à $560 \mathrm{~m} \mu$. La teneur en collagène total est déterminée en multipliant par 7,46 les valeurs en hydroxyproline obtenues (Neuman ef Logan, 1950 ; Denduchis et Mancini, 1967).

Expression des résultats. - Les résultats ont été exprimés par la moyenne des valeurs affectée de l'erreur type $\left(m \pm s_{m}\right)$. Dans certains cas, nous avons considéré les valeurs individuelles; nous avons alors établi des courbes de variations pour lesquelles nous avons calculé les corrélations et les paramètres de régression.

\section{Résultats.}

Evolution pondérale de la capsule festiculaire du Renard (tabl. 1).

Les variations pondérales de la capsule testiculaire sont représentées sur la figure 1 ; ces variations ont été comparées à celles du poids du testicule et du taux plasmatique de la testostérone.

La croissance pondérale de la capsule testiculaire du renard immature s'effectue en deux temps : la période impubère de croissance lente (pente de $0,76 \mathrm{mg} / \mathrm{j}$ ) est suivie de la période prépubère de croissance rapide (pente de $2,31 \mathrm{mg} / \mathrm{j}$ ). Cette croissance est en étroite corrélation avec l'époque de l'année $(P<0,01)$. La capsule atteint un poids maximum en janvier, à la puberté, puis décroît pour atteindre une valeur minimale qui 
se maintient sensiblement constante de mars-avril à septembre-octobre. A cette époque, le poids de la capsule croît de nouveau pour atteindre un maximum en janvier, à l'époque du rut.

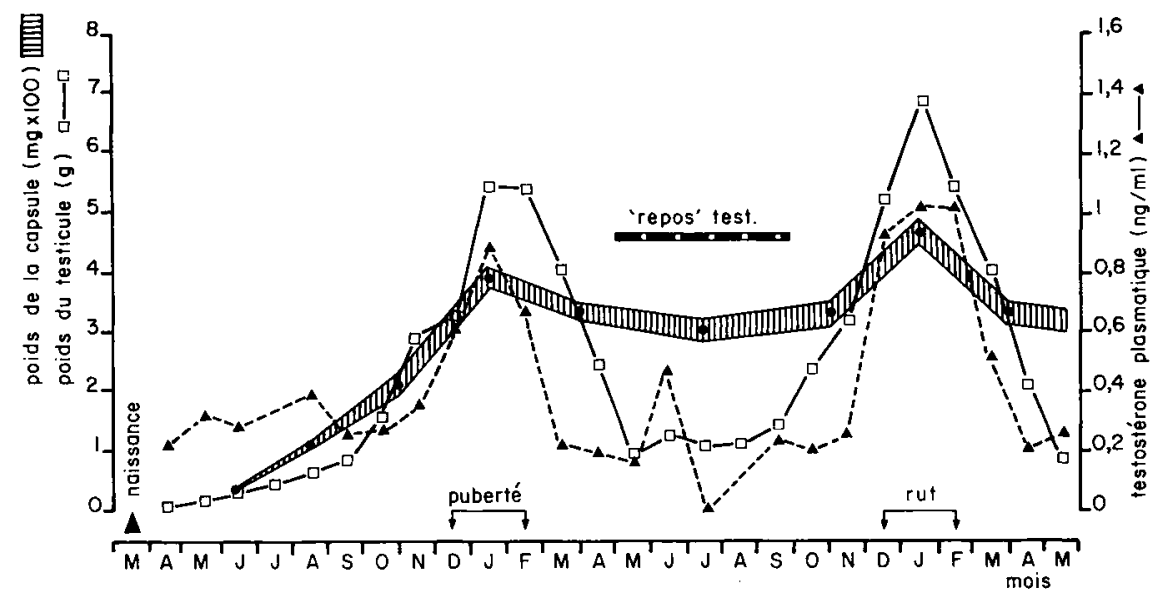

FIG. 1. - Variations saisonnières du poids frais de la capsule testiculaire, du poids du testicule et de la testostéronémie chez le renard immature et adulte (Capsule testiculaire : moyennes $\pm s_{\mathrm{m}}$ /voir tabl. 1. Testicule et testostérone : moyennes mensuelles).

Le poids de la capsule testiculaire est en corrélation étroite avec l'activité endocrine du testicule $(P<0,01)$ et varie en fonction du poids du testicule et de son activité spermatogénétique (fig. 2).

Un premier type de variations s'observe en période impubère ( $a$; pente de $128 \mathrm{mg} / \mathrm{g}$ ) pendant laquelle les gonocytes se multiplient. Un second type de variations est observé en période prépubère (b ; pente de $56 \mathrm{mg} / \mathrm{g}$ ) ; il y correspond une période de développement intense de la spermatogenèse, qui conduit à la production importante de spermatozoïdes en décembre-janvier. Chez l'adulte, en période de régression testiculaire comme en période de réveil testiculaire, le poids de la capsule est en étroife corrélation avec celui du testicule $(P<0,01)$, et ceci, quel que soit le stade de développement de la spermatogenèse ( $c$; pente de $32 \mathrm{mg} / \mathrm{g}$ ). Cette dernière pente est significativement différente de celle calculée en période prépubère chez l'animal immature $(P=0,05)$. Enfin, en période d'involution testiculaire maximale, pendant laquelle les tubes séminifères ne contiennent que quelques spermatocytes primaires au stade zygotène, le poids de la capsule est corrélé avec celui du testicule $(P<0,01)$ et ces couples de valeurs s'inscrivent normalement sur la droite de régression liée aux périodes de réveil et de régression testiculaires de l'adulte $(c+d$; pente de $31 \mathrm{mg} / \mathrm{g}$ ).

En période impubère la capsule testiculaire représente 15,5 p. $100 \mathrm{du}$ poids de la gonade. Au cours de la période prépubère ce rapport décroît et n'est plus que de 7,3 p. 100 lorsque l'animal est pubère. Chez l'adulte, c'est en période d'involution maximale que ce rapport est le plus élevé ; la capsule représente alors 26,4 p. 100 
du poids total du testicule. Ce rapport décroît de nouveau en période de réveil testiculaire et retourne à une valeur de 7,1 p. 100 à l'époque du rut.

Le poids sec et délipidé de la capsule présente les mêmes variations que le poids frais : augmentation jusqu'à la puberté chez le renardeau, minimum en période d'invoIution testiculaire maximale chez l'adulte $(P<0,01)$ et maximum en janvier, significativement différent de celui observé chez le renardeau pubère $(P<0,01)$.

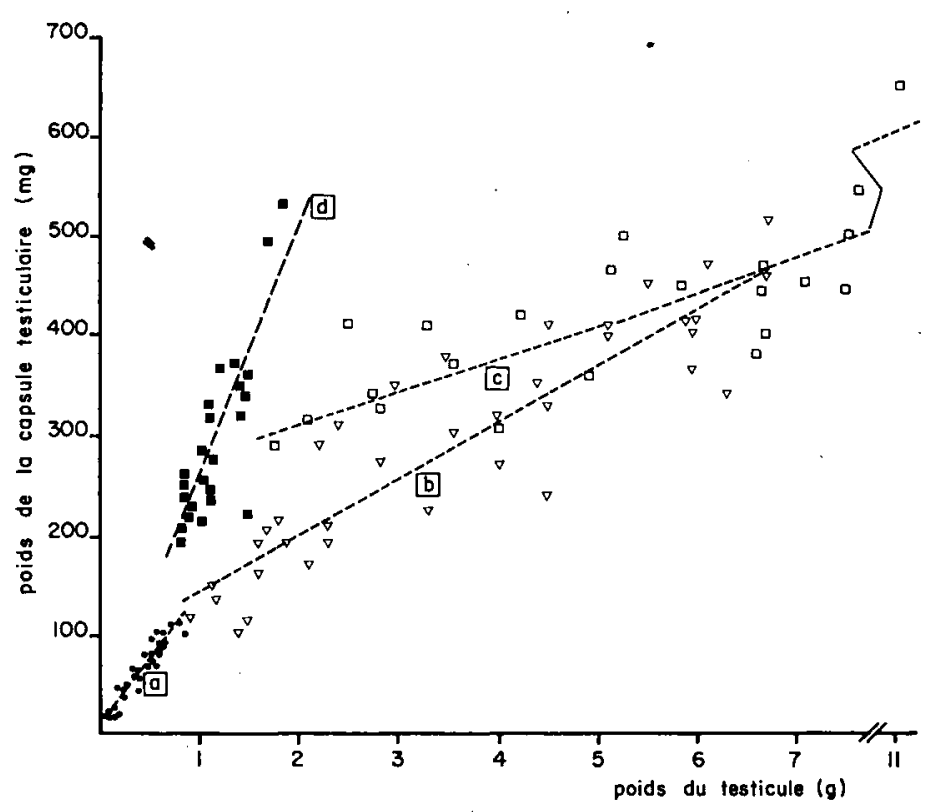

FIG. 2. - Relations poids frais de la capsule testiculaire et poids du testicule chez le renard impubère (O), prépubère $(\nabla)$, adulte en période de croissance et de régression testiculaire $(\square)$ et en période d'involution testiculaire maximale (0).

(Valeurs individuelles).

Equations des droites ( $y$ en $\mathrm{mg} ; x$ en $\mathrm{g}$ ).

$\begin{array}{llll}\square a & y=128 x+9,3 & r=0,94 & n=31 \\ \nabla b & y^{*}=56 x+89 & r=0,90 & n=37 \\ \square c & y^{*}=32 x+247 & r=0,84 & n=22 \\ \text { d } & y=240 x+15 & r=0,78 & n=24 \\ c+d & y^{*}=31 x+261 & r=0,78 & n=46\end{array}$

* Pente de b significativement différente de $c$; pente de $c+d$ non significativement différente de $c$ au seuil de 5 p. 100.

La feneur en lipides totaux de la capsule est faible (moins de 1 p. 100) ; le rapport poids sec et délipidé/poids frais traduit donc essentiellement l'état d'hydratation de cette enveloppe. Cette hydratation est maximale en période impubère et décroît progressivement pour atteindre un minimum en période prépubère $(P<0,01)$. Chez l'adulte ce rapport ne varie pas significativement au cours du cycle annuel. 


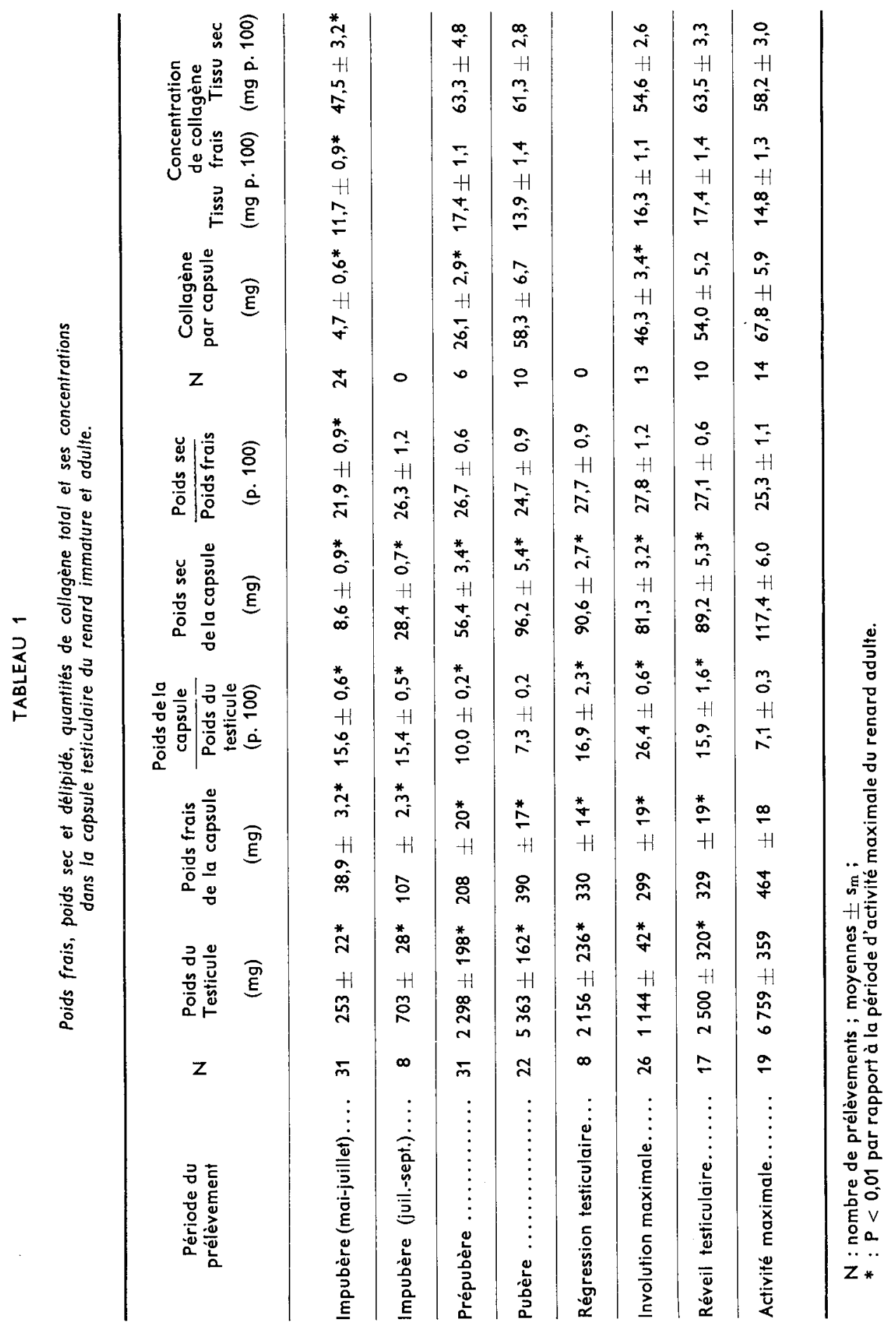


Evolution du collagène fotal de la capsule festiculaire du Renard (fabl. 1).

La teneur en collagène total évolue parallèlement au poids frais ou sec de la capsule. Chez le renardeau elle atteint un maximum à la puberté $(P<0,01)$; chez l'adulte elle passe successivement par un maximum à l'époque du rut et par un minimum en période d'involution testiculaire maximale $(P<0,01)$ (fig. 3$)$.

Chez le renardeau la concentration en collagène total de la capsule croît en fonction de l'âge, avec un maximum en période prépubère $(P<0,01)$; cette concentration varie peu au cours du cycle annuel chez l'adulte (fig. 3 ).
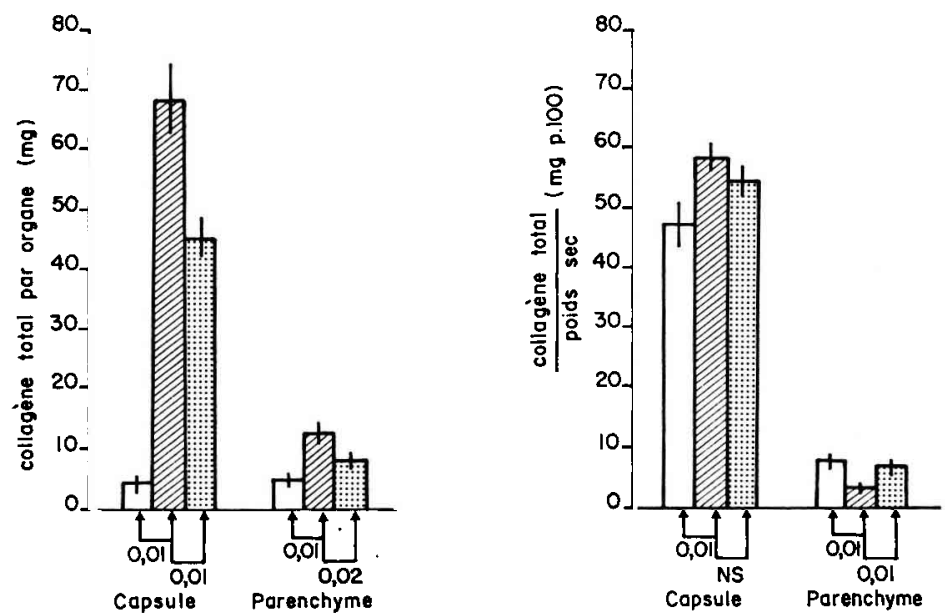

FIG. 3. - Collagène total et sa concentration dans la capsule* et le parenchyme** testiculaires du renard impubère ( $\square$ ), adulte et actif ( $\left.-1-s_{m}\right)$.

0,01 : signification de la différence

* valeurs du iableau 1

** pour des lots ef des poids de testicule respectivement de : (3), $581 \pm 22 \mathrm{mg} ;(10), 6195 \pm$ $141 \mathrm{mg}$; (14), $1121 \pm 70 \mathrm{mg}$.

Evolution du collagène total du parenchyme testiculaire du Renard (fig. 3).

Chez le renardeau la maturation testiculaire s'accompagne d'une hydratation tissulaire $(P<0,01)$ et d'un gain en collagène total $(P<0,01)$; par contre sa concentration décroît $(P<0,01)$.

Chez le renard adulte le parenchyme testiculaire se déshydrate au cours de la régression testiculaire $(P<0,01)$. En période d'involution testiculaire maximale, la teneur en collagène total est diminué $(P<0,02)$; par contre, sa concentration augmente $(P<0,01)$ et devient voisine de celle déterminée en période impubère chez l'animal immature (NS). 
Modifications histologiques de la capsule testiculaire du Renard (PI. I).

Variations de l'épaisseur de la capsule. - L'épaisseur de la capsule varie selon la zone de mesure et l'état fonctionnel de la gonade. Elle est plus mince à la face antérieure du testicule ef plus épaisse à la face postérieure (accolée à l'épididyme) ainsi qu'aux pôles. L'épaisseur varie au cours de la croissance pubertaire chez le jeune et pendant le cycle saisonnier de l'adulte. Elle est de 50 à $100 \mu$ chez l'animal impubère, de 75 à $150 \mu$ chez l'animal prépubère, de 100 à $200 \mu$ chez l'animal adulte en période d'activité testiculaire intense et de 200 à $500 \mu$ chez l'animal adulte en période d'involution testiculaire maximale.

Modifications histologiques de la capsule festiculaire. - Cefte étude a été abordée en utilisant la terminologie donnée par Davis, Langford et Kirby (1970).

A la tunica albuginea, qui occupe la quasi-totalifé de la capsule, est accolée à la face externe la tunica vaginalis viscérale, formée d'une seule couche de cellules (photos 2, 5). La tunica albuginea est essentiellement formée de fibroblastes et de fibres de collagène; son aspect varie au cours du cycle d'activité testiculaire. Chez le renardeau impubère, les fibroblastes sont très nombreux et réprésentés par des formes juvéniles au noyau plus ou moins ovale ; le collagène est encore peu abondant ef ne forme que des amas entre lesqueis on remarque un matériel amorphe PAS positif : des mucopolysaccharides (photo 2). Au cours de la maturation testiculaire chez l'animal immature, les fibroblastes diminuent en nombre ; leurs noyaux deviennent de plus en plus

\section{PLANCHE I}

Histologie de la capsule testiculaire du renard

(Coloration : trichrome de Masson)

PHOTO 1. - Renard impubère en juin ( $\times 158$ ). Dans la partie interne de la tunica albuginea $\left(t_{a}\right)$ transite une veine de gros calibre ( $v$ ) provenant du parenchyme testiculaire (P). La tunica albuginea émet un prolongement centripète - un septum $(S)$ - de structure identique.

PHOTO 2. - Renard impubère en juin (x 400). La tunica albuginea est formée de nombreux fibroblastes $(f)$, de mucopolysaccharides $\left(^{*}\right)$ et d'amas de collagène (c). Par cette tunique transitent les vaisseaux irriguant la capsule $\left(v_{c}\right)$. A sa partie externe est accolée la tunica vaginalis viscérale $\left(t_{v}\right)$.

PHОтО 3. - Renard adulte en janvier ( $\times 158$ ). A'la tunica albuginea est accolée une branche principale de l'artère festiculaire $(A)$.

PHОTO 4. - Renard adulfe en janvier ( $\times 400$ ). La funica albuginea est alors essentiellement formée de fibres de collagène coalescentes (c). Entre ces fibres on remarque quelques fibres de muscle lisse $(\mathrm{m})$ et quelques rares fibroblastes (f). Les mucopolysaccharides sont quasi absents.

PHOTO 5. - Renard adulte en juin (x 62). Une branche artérielle $(A)$ est maintenue à la funica albuginea par un épais faisceau de fibres de collagène $\left({ }^{*}\right)$.

PHOTO 6. - Renard adulte en juin (x 158). Les fibres de collagène sont rétractées et épaisses (c). Entre ces fibres on remarque quelques fibroblastes ( $f$ ) et, de nouveau, des mucopolysaccharides $\left({ }^{*}\right)$. 
fusiformes et denses. Progressivement, pendant cette période, les fibres de collagène s'organisent tandis que les mucopolysaccharides disparaissent (photo 4). En janvier, chez le pubère comme chez l'adulte, les mucopolysaccharides sont quasi absents et les fibres de collagènes sont nombreuses et coalescentes; quelques rares fibroblastes sont encore visibles entre ces fibres (photos 3,4 ) En période d'involution testiculaire maximale les fibres de collagène sont très rétractées et plus épaisses ; entre celles-ci on remarque à nouveau des mucopolysaccharides en grande quantité (photo 6).

Par la coloration du trichrome de Masson, Davis, Langford et Kirby (1970) ont différencié deux types de cellules dans la capsule testiculaire du Rat. Un premier type, les fibroblastes, représentés par leur forme mature au noyau fin et dense ; un second type, les fibres musculaires lisses, caractérisées par un noyau plus ou moins ovale ef une surface « en pointillés» (a stippled appearance). Ces deux types de cellules ont été retrouvés chez le Renard ; tous deux sont en nombre limité et disséminés entre les fibres de collagène (photo 4).

Les vaisseaux nourriciers de la capsule circulent dans la tunica albuginea (photos $2,3,4)$. En outre, accolés à la partie interne de cette tunique, on observe les vaisseaux irriguant le parenchyme testiculaire. Lorsqu'elles sont accolées à la capsule, les veines et les artères sont maintenues à la tunica albuginea par un faisceau de fibres de collagène qui s'épaissit en période de régression testiculaire (photos 3,5 ).

Les septa sont des prolongements centripètes de la funica albuginea ; à leur base on rencontre très fréquemment des vaisseaux de gros calibre destinés à l'irrigation du parenchyme (photo 1). En période impubère ces cloisons renferment de nombreux fibroblastes de type juvénile (photos 1,7 ) et manifestent une très forte réaction PASpositive (photo 8). A la puberté et chez l'animal adulte en pleine activité, les septa ont une structure fibreuse ; on y observe alors des fibres de collagène. Une telle structure

PLANCHE 2

Histologie du tissu intertubulaire du festicule de renard

(Colorations : 7-Hémalun-Picro-Indigocarmin. 8 à 10 : PAS-Hématoxyline)

PHOTO 7. - Renord impubère en juin (x 300). Le tissu intertubulaire renferme alors de nombreux fibroblastes ( () : certains sont tangeants à la membrane basale des fubes séminifères ( $m b$ ). Un septum (S) sépare les tubes séminifères ; il est formé de fibroblastes et de quelques fibres de collagène.

PHOTO 8. - Renard impubère en juin (x 250). Par les septa transitent les vaisseaux ( $v$ : veine ; $a$ : artériole) irriguant le parenchyme. Parmi les septa on remarque quelques îlots de cellules de Leydig (L). Pendant cette période la membrane basale est fine (mb).

PHOTO 9. - Renard adulte en janvier (x 200). Les fibroblastes ont disparu ; ils ont fait place à une trame fibreuse $\left(^{*}\right)$ qui entoure les tubes séminifères, les îlołs de cellules de Leydig (L), les capillaires péritubulaires $\left(c_{p}\right)$ et intertubulaires $\left(c_{1}\right)$.

PHOTO 10. - Renard adulte en juin (x 200). Pendant cette période la membrane basale est épaissie $(\mathrm{mb})$; les septa qui donnent les graines fibreuses périvasculaires sont eux aussi très épaissis (*). 

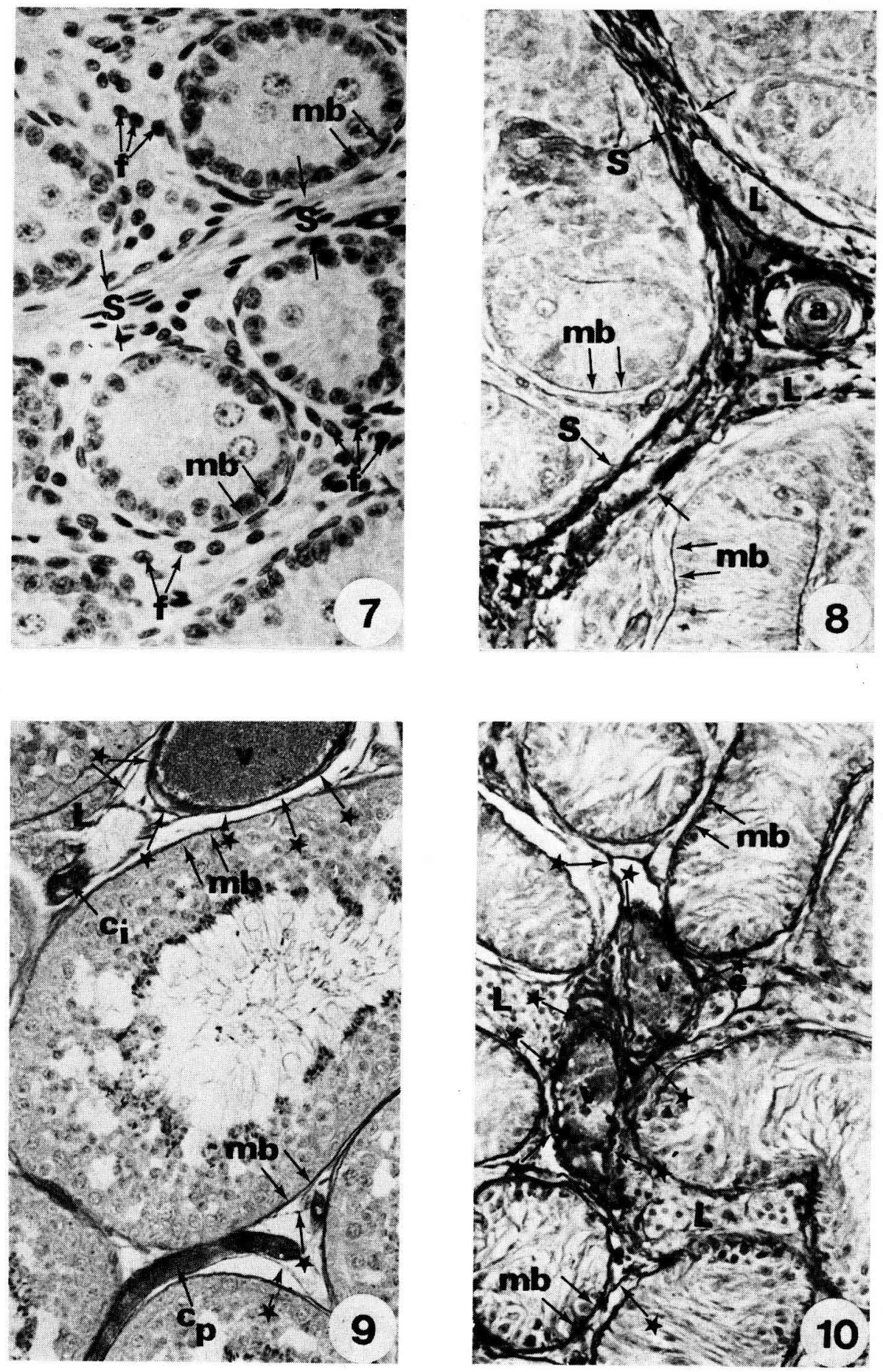
fait que ces cloisons sont très souvent déchiquetées, voire même absentes des préparations, après section des organes par le microtome. Par ces septa transitent la plupart des vaisseaux qui irriguent le parenchyme testiculaire. En période de pleine activité la gaine fibreuse que forment les septa autour des vaisseaux est peu épaisse (photo 9). Au cours de la période de régression testiculaire ces gaines s'épaississent et parallèlement se produit la déformation des vaisseaux qui transitent par eux (photo 10); à un fort grossissement on remarque alors la présence de mucopolysaccharides dans ces gaines.

Une trame fibreuse intertubulaire existe dans le tissu interstitiel du renard adulte (photo 9) ; elle présente une maturation au cours de la croissance pubertaire du renardeau. Chez l'animal impubère cette trame renferme essentiellement des fibroblastes de type immature (photos 1,7 ) ; parmi ceux-ci on observe quelques fibres ainsi que du matériel amorphe. En octobre, on observe la distension des fubes séminifères et l'apparition d'une trame fibreuse très lâche; à cette époque les espaces interiubulaires sont imporfants. Au cours de la période prépubère ces espaces intertubulaires diminuent, les fibroblastes disparaissent et font place à la trame fibreuse, PAS positive, visible chez l'animal adulte (photo 9) ; celle-ci entoure les îlots de cellules de Leydig, les tubes séminifères el quelques vaisseaux de petit diamètre, essentiellement les capillaires, qui ne transitent pas par les septa. Cette trame ne montre pas de variations sensibles au cours du cycle annuel de l'adulte. D'autre part, on constate que les nombreux fibroblastes immatures, disposés circulairement autour des tubes séminifères de l'animal impubère (photo 7), ne sont plus présents chez l'animal actif (photo 9). Enfin, on remarque, qu'au cours de la période de régression testiculaire chez l'adulte, la membrane basale des tubes est fortement épaissie (photo 10).

\section{Discussion.}

10 L'analyse histologique de la capsule testiculaire des mammifères a permis de constater la structure tristratifiée de cette enveloppe chez toutes les espèces étudiées (Leeson ef Adamson, 1962 ; Mancini ef al., 1964 ; Leeson et Cokson, 1974). Seules la tunica vaginalis viscérale et la tunica albuginea ont été observées chez le Renard ; la tunica vasculosa, généralement très mince, n'a pu être dissociée ici de la tunica albuginea. Cette analyse a permis de constater l'importance relative de la tunica albuginea ainsi que sa structure, formée essentiellement de fibres de collagène et de fibroblastes. En outre, quelques fibres de muscles lisses ont pu être mises en évidence ; mais contrairement à ce qui a été observé chez le Lapin, le Rat, le Chien, le Chat et l'Homme, elles n'ont jamais été rencontrées groupées en faisceaux (Davis, Langford ef Kirby, 1970 ; Langford et Heller, 1973 ; Leeson et Cokson, 1974).

La structure des septa est semblable à celle décrite chez l'Homme à l'état adulte (Mancini ef al., 1964) ; ils sont essentiellement formés de fibres de collagène. La trame fibreuse infertubulaire est présente dans le testicule de nombreuses espèces de mammifères adultes ; pour Hooker (1970), Sniffen (1950) et Mancini et al. (1964) cette trame est formée de réticuline tandis que pour Fawcett, Neaves et Flores (1973) elle est constituée de collagène, deux protéines de composition chimique très voisine (Ganter ef Jolles, 1970). 
Lors de la croissance puberfaire du renardeau, l'ensemble capsule-septa-tissu fibreux intertubulaire présente les mêmes transformations que celles décriles chez l'Homme par Mancini ef al. (1964) : épaississement de la capsule, disparition de nombreux fibroblastes et des mucopolysaccharides, enrichissement progressif de la tunica albuginea et des septa en collagène, enfin, enrichissement du tissu intertubulaire en protéines fibreuses.

L'épaississement réversible de la capsule au cours du cycle d'activité saisonnier du testicule a déjà été signalé chez le Campagnol (Clarke et Forsith, 1964), l'Ecurevil (Delost, 1966) el le Furet (Ishida, 1968) ; chez ces trois espèces ainsi que chez le Renard, cet épaississement s'accompagne d'une rétraction des fibres de collagène. Mais la réapparition des mucopolysaccharides dans ces structures, signe d'une synthèse faible ou ralentie de collagène (Dunphy ef Udupta, 1955), ainsi que la rétraction et l'épaississement des septa en période de régression testiculaire n'ont pas été rapportés auparavant.

$2^{\circ}$ Le poids relatif de la capsule testiculaire du renardeau $(15,5$ p. 100$)$ est supérieur à celui du rat impubère (8,3 p. 100, Denduchis et Mancini, 1967) et de l'Agneau (11,4 p. 100, Courot, 1971). Ce poids est plus élevé chez le renard actif que chez le Rat adulte (3,6 p. 100 Denduchis et Mancini, 1967) et chez le Bélier (5,4 p. 100, Courol, 1971) ; il est très voisin de celui du Verrat (6 à 7,7 p. 100, Kennely et Foote, 1964 ; Swierstra et Rahnefeld, 1967).

La croissance pondérale de la capsule testiculaire au cours de la période prépubère a déjà été signalée chez le Rat et chez l'Agneau (Denduchis et Mancini, 1967 ; Courot, 1971). Mais, aucune variation pondérale de cette enveloppe n'a été remarquée auparavant chez l'animal adulte, au cours du cycle saisonnier ou après involution provoquée de la gonade. Les relations qui existent entre le poids de la capsule et celui du testicule (fig. 2) permettent de conclure que ces variations sont lentes, progressives et synchrones des modifications qui se produisent dans le parenchyme.

$3^{\circ}$ Les concentrations en collagène total de la capsule et du parenchyme, chez le Renard adulte et actif, sont voisines de celles du Rat adulte (Denduchis et Mancini, 1967) ; par contre sa concentration dans le parenchyme est inférieure à celle de l'Homme (Denduchis, Gonzales et Mancini, 1972) et ceci, malgré une structure du testicule du Renard plus voisine de celle de l'Homme que de celle du Rat.

Le collagène total de la capsule testiculaire du Renard augmente en valeurs absolue et relative, au cours de la croissance pubertaire. Ces résultats sont en concordance avec les données de l'analyse histologique et confirment ceux obtenus chez le Rat par Denduchis et Mancini (1967). Enfin, la concentration de cette protéine augmente avec l'âge dans la capsule comme dans la peau (Gonzales-Cadavit et al., 1963) ef de nombreux autres tissus (pour revue : Denduchis ef Mancini, 1967).

Le collagène total du parenchyme testiculaire du Renard augmente lors de la maturation testiculaire ; par contre sa concentration diminue très nettement. Ces résultats sont conformes à ceux obtenus chez le Rat (Denduchis et Mancini, 1967) et incitent donc à conclure que le collagène total du parenchyme évolue différemment de celui de la capsule en fonction de l'âge. Les données de l'histologie et celles de la bibliographie montrent que le collagène du parenchyme est principalement associé aux septa, aux parois des artères, à la membrane basale des tubes séminifères ainsi qu'aux 
fibres du tissu intertubulaire (Leeson et Leeson, 1963 ; Mancini et al., 1964 ; Fawcett, Neaves et Flores, 1970 ; Bustos-Obregon el Courot, 1974 ; de Krester, Kerr et Paulsen, 1975). Or ces structures ne représentent qu'un faible compartiment testiculaire (moins de 1 p. 100) et ne peuvent donc présenter que de faibles variations par rapport à celles subies par l'ensemble du parenchyme. De ce fait, nous pensons qu'au cours de la maturation testiculaire, qui entraîne essentiellement le développement des strucfures pauvres en collagène, non seulement la quantité de cette protéine augmente dans les structures qui en sont riches, mais aussi sa concentration ; ce dernier point ne pouvant apparaître sur la figure 3 étant donné que les concentrations sont ici calculées par rapport à l'ensemble du parenchyme. Deux remarques confirment ceci : l'aspect des septa évolue parallèlement à celui de la tunica albuginea et, d'une manière générale chez les espèces étudiées, la membrane basale des tubes séminifères présente une maturation avec, entre autre phénomène, un enrichissement en collagène (Leeson ef Leeson, 1963 ; Ross, 1967 ; Bressler et Ross, 1972 ; Mancini ef al., 1964 ; BurgosObregon et Courot, 1974). Ainsi, le collagène des structures parenchymateuses du testicule riches en cette protéine présente avec l'âge les mêmes variations que celui de la capsule.

Chez le Renard adulte, le collagène total de la capsule et du parenchyme décroît significativement au cours de la période de régression testiculaire (fig. 3). Il ressort d'autre part que les variations des compartiments parenchymateux et capsulaires riches en collagène sont synchrones des variations de la spermatogenèse et de l'hormogenèse. Ceci suggère donc que les transformations des structures fibreuses du testicule constituent un élément important de l'activité testiculaire et qu'elles sont probablement sous la dépendance des hormones gonadotropes hypophysaires réglant le cycle d'activité saisonnière, soit directement, soit indirectement par l'intermédiaire de la testostérone. De nombreuses hormones sont par ailleurs capables de stimuler ou d'inhiber la synthèse du collagène (pour revue : Laitinen, 1967). De plus ces résultats sont à rapprocher de ceux obtenus après hypophysectomie, dans le derme du Rat (Fabianek ef Herd, 1966) et dans le parenchyme testiculaire de l'Homme (Denduchis, Gonzales ef Mancini, 1972). Dans les deux cas, cette opération entraîne la diminution du collagène total et, dans le cas du testicule humain, l'apparition d'un matériel amorphe à la périphérie des tubes séminifères. Dans ce dernier cas, il a pu être montré que le collagène testiculaire augmentait après l'injection de HCG, alors qu'il restait à une valeur faible après l'injection de testostérone ou d'HMG.

$4^{\circ}$ Récemment (Joffre, 1976a), nous avons pu remarquer que, chez le Renard la reprise d'activité automnale chezl'adulte s'effectuait selon des modalités très voisines de celle de la croissance pubertaire de l'animal immature ; un tel résultat nous avait fait conclure à une « puberté annuelle» chez l'adulte. II ressort de la présente étude que la croissance pondérale de la capsule du jeune et de l'adulte s'effectue selon des modalités différentes ; les deux pentes de croissance sont en effet significativement différentes (fig. 2). Néanmoins, on peut constater qu'au cours de ces deux périodes de croissance un enrichissement progressif en collagène des tissus se produit avec, simultanément, la disparition des mucopolysaccharides. Ceci tend donc à prouver que les mécanismes endocrininens impliqués dans ces deux types de croissance sont de même nature, mais d'ampleur différente ; il est à noter qu'à la puberté le poids de la capsule, ainsi que 
celui du testicule entier, est significativement différent de celui atteint par l'animal adulte en janvier. Enfin, des différences importantes avaient été observées entre l'état impubère de la gonade et son état de « repos» chez l'adulte. Les résultats obtenus, tant au niveau du tissu intertubulaire qu'à celui de la capsule confirment cette différence.

\section{Significations physiologiques de ces transformations.}

a) Ces résultats démontrent que la rétraction de la capsule en période de régression testiculaire, chez le Renard adulte, est un phénomène actif. Pendant cette même période, on a pu remarquer la spiralisation des artères et des artérioles, ainsi que la distorsion des veines du parenchyme testiculaire (Joffre et Kormano, 1975). Or, l'analyse histologique montre les liens étroits qui unissent la capsule ef les septa aux vaisseaux de gros calibre destinés au parenchyme testiculaire. Dès lors, il est possible d'envisager l'intervention directe de la capsule et des septa sur les modifications lentes, progressives et cycliques de la trame vasculaire irriguant le testicule : la rétraction active de la capsule, qui n'est qu'un aspect particulier de l'involution testiculaire, contraindrait les vaisseaux pris dans la tunica albuginea et ses septa à suivre cette involution, soit en se spiralisant (cas des vaisseaux résistants), soit en se déformant (cas des veines) ; les capillaires échappant à cette déformation (Joffre, 1976b) du fait de la seule présence de quelques fibres en nombre très limité. II est à remarquer que, chez le Rat, cette organisation vasculaire n'existe pas ; les septa sont absents et le transit des artères dans la capsule se limite à leur entrée dans le testicule (Kormano, 1967a). Or, chez cette espèce, l'involution provoquée du testicule, soit par l'hypophysectomie (Harrison, 1949), soit par la cryptorchidie (Kormano, 1969) ne provoque pas de déformation des vaisseaux, ceux-ci se contentant de subir une réduction de taille qui laisse inchangée la configuration de la trame vasculaire.

Dès lors, on peut penser que la capsule testiculaire du Renard adulte intervient dans le contrôle saisonnier de l'hémodynamique testiculaire. Sa rétraction active, responsable de la spiralisation des vaisseaux résistants, entraînerait la diminution du débit sanguin capillaire du testicule au cours de la période de régression testiculaire (Joffre et Joffre, 1973) par diminution de la pression sanguine à l'entrée du capillaire ; on sait en effet que la présence d'artères spiralées entraîne la diminution de cette pression (Moll et Kunzel, 1971, 1973).

b) Une barrière (blood testis barrier) limite la pénétration de certaines substances dans les tubes séminifères des mammifères adultes (Setchell, Voglmayr et Waites, 1969 ; Johnson, 1970 ; Koskemies, Kormano et Alfthan, 1973). Les recherches tendant à localiser cette barrière ont permis de situer celle-ci, pour une part importante, au niveau des jonctions entre cellules de Sertoli, et, pour une part non négligeable, au niveau de la membrane basale (Ross, 1970 ; Kormano, 1970 ; Dym et Fawceft 1970 ; Fawcett, Leak et Heidger, 1970). De nombreuses observations permettent de relier l'apparition de cette barrière chez l'animal immature (Kormano, 1967b ; Vitale, Fawcett et Dym, 1973) à la maturation de la membrane basale et, tout particulièrement à son enrichissement en collagène (Leeson et Leeson, 1963 ; Mancini et al., 1964 ; Ross, 1967 ; Bustos-Obregon et Courot, 1974) ; la relation directe entre le collagène ef l'efficacité de la barrière restant à prouver. 
Dans ces conditions, il est possible d'envisager un lien direct entre les modifications histologiques et biochimiques de la membrane basale du tube séminifère ef la mise en place de la «blood testis barrier» chez le renardeau.

D'autre part, poursuivant celte analyse, il est possible d'envisager les transformations du tissu fibreux, observées chez l'animal adulte au cours du cycle annuel, en liaison avec une éventuelle modification de l'efficacité de cette barrière. Toutefois, dans ce cas, il est fort probable que les variations observées n'impliquent nullement de variations de cette barrière. On sait en effet que, si les variations ce structure de la basale apparaissent hormonodépendantes chez la plupart des mammifères, soit à l'état impubère, soit à l'état adulte après hypophysectomie (Courot, Hochereau-de Reviers et Ortavant, 1970 ; Bressler ef Ross, 1972 ; Hovatta. 1972), la mise en place de cetfe barrière, par contre, et, plus tard, sa variation d'efficacité ne sont pas profondément modifiées par l'hypophyse et ses hormones (Setchell, Voglymar et Waites, 1969 ; Johnson, 1973 ; Vitale, Fawcett ef Dym, 1973).

Néanmoins, étant donné que le renard présente sur bien des points une physiologie testiculaire différente de celle des mammifères domestiques ou de laboratoire (Joffre, 1976b), en relation avec une activité saisonnière très prononcée, cette hypothèse de travail ne peut être négligée. Dès lors, il convient d'examiner la structure fibreuse de la membrane du fube séminifère et d'envisager la détermination simultanée de paramètres permettant de quantifier l'efficacité de cette«blood testis barrier».

Reçu en décembre 1976. Accepté en février 1977.

Ce travail fait l'objet d'un chapitre de la Thèse de Doctorat ès-Sciences no CNRS AO 10796, Université de Poitiers.

\section{Références}

BLOMFIELD J., FARRAR J. F., 1964. Factors affecting the determination of hydroxyproline. Anal. Chem., 36, 950-952.

BRESSLER R. S., ROSS M. H., 1972. Differentiation of peritubular myoid cells of the testis : Effects of intratesticular implantation of newborn mouse testes into normal and hypophysectomized adults. Biol. Reprod., 6, 148-159.

BUSTOS-OBREGON E., COUROT M., 1974. Ultrastructure of lamina propria in the ovine seminiferous tubule. Cell Tiss. Res., 150, 481-492.

CLARKE J. R., FORSITH I. A., 1964. Seasonal changes in the gonads and accessory reproductive organs of the vole Microtus agrestis. Gen. comp. Endocr., 4, 233-242.

COUROT M., 1971. Ełablissement de la spermatogenèse chez l'agneau (Ovis aries). Ełude expérimentale de son contrôle gonadotrope ; importance de lo lignée serfolienne. Thèse doct. ès-Sci., Paris, C.N.R.S. No A.O. 6317.

COUROT M., HOCHEREAU-de REVIERS M. T., ORTAVANT R., 1970. Spermatogenesis. In A. D. JOHNSON, W. R. GOMES, N. L. Van DEMARK, The testis. Vol. I, 339-442, Acad. Press, N.-Y., London.

CREED R. F. S., 1960. Observations on reproduction in the wild red fox (Vulpes vulpes). An account with special reference to the occurrence of fox dog crosses. Brit. vef. J., 116, 419-426.

DAVIS J. R., LANGFORD G. A., KIRBY P. J., 1970. The testicular capsule. In A. D. JOHNSON, W. R. GOMES, N. L. Van DEMARK, The festis. Vol. I, 281-337, Acad. Press, N.-Y., London. 
DELOST P., 1966. Reproduction et cycles endocriniens de l'écureuil. Arch. Sc. physiol., 20, 425-456.

DENDUCHIS B., GONZALES N., MANCINI R. E., 1972. Concentration of hydroxyproline in testes of hypophysectomized patients before and after treatment with gonadotrophins and testosterone. J. Reprod., Fert., 31, 111-114.

DENDUCHIS B., MANCINI R. E., 1967. Quantitative variations in total and soluble collagen in festes of perpubertal and adult rats. Endocrinology, 30, 1163-1167.

DUNPHY J. E., UDUPA K. N., 1955. In JACKSON D. S., 1957. Connective tissue growth stimulated by carrageenin. Biochem J., 65, 277-284.

DYM M., FAWCETT D. W., 1970. The blood festis barrier in the rat and the physiological compartmentation of the seminiferous epithelium. Biol. Reprod., 3, 308-326.

FABIANEK J., HERD A., 1966. Glycoprotein and collagen metabolism in the dermis of hypophysectomized rats. Endocrinology, 78, 1255-1258.

FAWCETT D. W., LEAK L. V., HEIDGER P. M., 1970. Electron microscopic observations on the structural components of the blood-testis barrier, J. Reprod. Fertil., Suppl. 10, 101-115.

FAWCETT D. W., NEAVES W. B., FLORES M. N., 1973. Comparative observations on the intertubular lymphatics and the organization of the interstitial tissue of the mammalian testis. Biol. Reprod., 9, 500-532.

GANTER P., JOLLES G., 1970. Histochimie normale et pathologique, Vol 2, Gauthier-Villars, Paris.

GONZALES-CADAVIT N. G., DENDUCHIS B., MANCINI R. E., 1963. Soluble collagens in normal skin, from embryo to adulthood. Lab. Invest., 12, 598-605.

HARRISON R. G., 1949. The comparative anatomy of the blood supply of the mammalian testis. Proc. Zool. Soc. Lond, 119, 325-344.

HOOKER C. W., 1970. The intertubular tissue of the testis. In A. D. JOHNSON, W. R. GOMES, N. L. Van DEMARK. The testis. Vol. I, 483-550, Acad. Press, N.-Y., London.

HOVATTA O.; 1972. Effect of androgens and antiandrogens on the development of the myoid cells of the rat seminiferous tubules (organ culture). Z. Zellforsch., 131, 299-308.

ISHIDA K., 1968. Age and seasonal changes in the testis of mink. Arch. Histol. jap., 29, 193-205.

JOFFRE J., JOFFRE M., 1973. Seasonal changes in the testicular blood flow of seasonal breeding mammals : dormouse (Glis glis), ferret (Mustella furo) and fox (Vulpes vulpes). J. Reprod. Fertil., 34, 227-233.

JOFFRE M., 1976a. Puberté et cycle génital saisonnier du renard mâle (Vulpes vulpes). Ann. Biol. anim. Biochim. Biophys., 16,, 503-520.

JOFFRE M., 1976b. Débit sanguin capillaire du testicule chez le renard roux (Vulpes vulpes). Relation ovec l'activité testiculaire pendant la période prépubère ef au cours du cycle saisonnier. Thèse Doct. ès-Sci., Poitiers, No C.N.R.S. A.O. 10796.

JOFFRE M., JOFFRE J., 1975. Variations de la testostéronémie au cours de la période prépubère du renardeau et du cycle génital saisonnier du renard mâle adulte. $C$. R. Acad. Sci., Paris, Série D, 281, 819-821.

JOFFRE M., KORMANO M., 1975. An angiographic study of the fox testis in various stages of sexual life. Anat. Rec., 183, 599-604.

JOHNSON M. H., 1970. The pituitary and the blood-testis barrier. J. Reprod. Ferifl., 22, 181-182.

JOHNSON M. H., 1973. The rôle of the pituitary in the development of the blood-testis barrier in mice. J. Reprod. Fertil., 32, 509-511.

KENNELY J. J., FOOTE, R. H., 1964. Sampling boar testes to study spermatogenesis quantitatively and to predict sperm production. J. anim. Se., 23, 160-167.

KORMANO M., 1967a. An angiographic study of the testicular vasculature in the post-natal rat. $Z$ Anat. Entwickl.-Gesch., 126, 138-153.

KORMANO M., 1967b. Dye permeability and alkaline phosphatase activity of testicular capillaries in the postnatal rat. Histochemie, 9, 327-338.

KORMANO M., 1969. Microvascular supply of the regenerative rat testis following cadmium injury. Virchows Arch. Abat. A. Pagth. Ant, 349, 229-235.

KORMANO M., 1970. The development and function of the peritubular tissue in the rat testis. Morphol. Aspects Androl., 1, 86-89.

KOSKEMIES A. I., KORMANO M., ALFTHAN O., 1973. Proteins of the seminiferous tubule fluid in man. Evidence for a blood testis barrier. J. Reprod. Fertil., 32, 79-86. 
KRESTER D. M. de, KERR J. B., PAULSEN C. A., 1975. The peritubular tissue in the normal an pathological human testis. An ultrastructural study. Biol. Reprod., 12, 317-324.

LAITINEN O., 1967. The metabolism of collagen and its hormonal control in the rat. Acta endocr., (Kbh)., Suppl. 120, 1-86.

LANGFORD G. A., HELLER C. G., 1973. Fine structure of the muscle cells of the human testicular capsule : basis of the testicular contractions. Science, N.-Y., 179, 573-575.

LEESON T. S., ADAMSON L., 1962. The mammalian tunica vaginalis of testis : its fine structure and function. Acta anat., 51, 226-240.

LEESON T. S., COKSON F. B., 1974. The mammalian testicular capsule and its muscle elements. J. Morphol., 144, 237-254.

LEESON E. S., LEESON T. S., 1963. The postnatal development and the differentiation of the boundary tissue of the seminiferous tubule of the rat. Anat. Rec., 147, 243-260.

LEYMARIE P., STRAUSS N., SCHOLLER R., 1974. Dosage radioimmunologique rapide de la testostérone plasmatique chez l'adulte et l'enfant. Vérification de la spécificité en spectrométrie de masse. Path. Biol., 22, 877-882.

MANCINI R. E., VILLAR O., PEREZ del CERRO M., LAVIERI J. C., 1964 . Changes in the stromal connective tissue of the human testis. A histological, histochemical and electromicroscopical study. Acta Physiol. lat.-amer., 14, 382-391.

MARTIN J., AXELROD A. E., 1963. A modified method for determination of hydroxyproline. Proc. Soc. exp. Biol. Med., 83, 461-462.

MOLL W., KUNZEL W., 1971. Blood pressure in the uterine vasculature system of ancesthetized pregnant guinea pigs.. Pflügers Arch., 330, 310-322.

MOLL W., KUNZEL W., 1973. The blood pressure in arteries entering the placenta of guinea pigs, rats, rabbits and sheep. Pflügers Arch., 338, 125-131.

NEUMAN R. E., LOGAN M. A., 1950. The determination of collagen and elastin in tissues. J. biol. Chem., 186, 549-556.

ROSS M. H., 1967. The fine structure and development of the péritubular contractile cell component in the semineferous tubules of the mouse. Am. J. Anat., 121, 523-558.

ROSS M. H., 1970. The Sertoli cell and the blood-testis barrier : an electronmicroscopic study. Morphol. Aspects Androl., 1, 83-86.

ROWLANDS I. W., PARKES A. S., 1935. The reproductive processes of certain mammals. VIII. Reproduction in foxes (Vulpes SpP). Proc. zool. Soc., London, 823-841.

SETCHELL B. P., VOGLMAYR J. K., WAITES G. M. H., 1969. A blood-testis barrier restricting passage from blood into rete testis fluid but nof into lymph. J. Physiol., London, 200, 73-85.

SNIFFEN R. C., 1950. In HOOKER C. W., 1970. The intertubular tissue of the testis. In A. D. JOHNSON, W. R. GOMEZ, N. L. VanDEMARK, The testis, Vol. 1, 483-550, Acad. Press. N. Y., London.

SWIERSTRA E. E., RAHNEFELD G. W., 1967. Semen and testis characteristics in young Yorkshire and Lacombe boars. J. anim. Sci., 26, 149-157.

VIALE R., FAWCETT D. W., DYM M., 1973. The normal development of the blood-testis barrier and the effects of clomiphene and estogen treatment. Anot. Rec., 176, 333-344. 